\title{
Chronic interstitial pneumonitis in end-stage sarcoidosis
}

\section{To the Editors:}

End-stage sarcoidosis is typically characterised by severe interstitial fibrosis occurring along the bronchovascular bundles with cystic changes $[1,2]$. Chronic interstitial pneumonitis typically occurs in the early stages of sarcoidosis and is localised to the areas affected by granulomas [3-5]. Herein, we report end-stage sarcoidosis patients who underwent lung transplantation at our centre (University of Southern California, Keck School of Medicine, Los Angeles, CA, USA) and who surprisingly had evidence of moderate-to-severe chronic interstitial pneumonitis, which was previously considered atypical in end-stage sarcoidosis. Furthermore, two patients had a pattern resembling usual interstitial pneumonitis (UIP) with fibroblastic foci.

We reviewed the charts and microscopic slides of all sarcoidosis patients who underwent lung transplantation at the University of Southern California. Patients were only included if the initial biopsy reports used to support the diagnosis contained descriptions compatible with a diagnosis of sarcoidosis and/or met the American Thoracic Society/ European Respiratory Society/World Association of Sarcoidosis and Other Granulomatous Disorders criteria [6].

All microscopic slides from the explanted lungs and mediastinal/hilar regional lymph nodes from these patients were examined by two pathologists (J.M. Oblad and M.N. Koss) who were unaware of any clinical information except for the history of lung transplantation for sarcoidosis. The pathologists were asked to grade the average granuloma burden (lung and lymph nodes) and interstitial fibrosis (lung) individually as follows. Grade 0 (absent): no granulomas or fibrosis detected in the specimen; Grade 1 (mild): less than two granuloma per low-power field (lpf) or occasional foci of fibrosis per low lpf; Grade 2 (moderate): two to five granuloma per lpf or $<50 \%$ of parenchyma with fibrosis per lpf; and Grade 3 (severe): more than five granuloma per lpf or $>50 \%$ of parenchyma with fibrosis $(\mathrm{lpf}=40 \times$ magnification). Specifically, for average granuloma burden, the total number of granulomas in four random lpf were divided by four with the result graded using the system above. Similarly, the average grade of fibrosis was calculated by dividing the total grade of fibrosis in four random lpf by four. Interstitial pneumonitis was defined as interstitial infiltration by lymphocytes and/or plasma cells. The pathologists were then asked to comment on the likely histological diagnosis.

Seven subjects, five females and two males, were enrolled in the study. Clinical characteristics with associated histological findings are described in table 1 . The mean (range) age at lung transplantation was 53.7 (43-66) yrs and all subjects had moderate to severe grades of interstitial fibrosis. Three of the seven subjects (subjects 1-3; 42.9\%) had fibrotic granulomatous lung disease with no significant interstitial pneumonitis, which is consistent with end-stage sarcoidosis (fig. 1a). The remaining four subjects (subjects $4-7 ; 57.1 \%$ ) had evidence of moderate-to-severe chronic interstitial pneumonitis atypical of end-stage sarcoidosis (fig. 1b). Furthermore, two of the subjects with chronic interstitial pneumonitis (subjects 6 and 7; $28.6 \%$ ) had fibroblastic foci and patchy distribution of the pneumonitis, findings that are consistent with a UIP pattern (fig. 1c and d).

Interstitial pneumonitis typically occurs in early stages of sarcoidosis and is usually mild [3-5]. Specifically, in a previous study, $62 \%$ of 128 open lung biopsy specimens from patients with sarcoidosis in its early stages showed mild cellular interstitial pneumonitis as the predominant histological finding, suggesting that granuloma formation in early sarcoidosis is preceded by interstitial pneumonitis [7]. Furthermore, the interstitial pneumonitis or alveolitis decreased as sarcoidosis resolved or progressed into chronic disease [7, 8]. Unexpectedly, four of the patients (subjects 4-7; 57.1\%) showed moderate to severe diffuse chronic interstitial pneumonitis. Although diffuse interstitial pneumonitis is considered to be rare in sarcoidosis, we found similarities with one case of sarcoidosis reported by AISNER et al. [9]. The authors presented a sarcoid patient with diffuse interstitial pneumonitis and honeycomb changes as the predominant feature with only focal aggregates of non-necrotising granulomas in the lung. Furthermore, the areas of granuloma burden were not associated with diffuse interstitial pneumonitis, in contrast to findings by other authors $[5,7]$. In this particular case, much as in our cases, the presence of granulomas in the local mediastinal lymph nodes in combination with the clinical presentation supported the diagnosis of sarcoidosis. Unfortunately, the type of interstitial pneumonitis in this case is not well defined. However, it is plausible to postulate that there is a subset of patients with sarcoidosis who have significant diffuse interstitial pneumonitis in the lungs in later stages of the disease and who may demonstrate a different clinical course when compared with most sarcoidosis patients.

Unexpectedly, two of these patients (subjects 6 and 7) had a pattern indistinguishable from UIP pattern (fig. 1d), with fibroblastic foci present. The regional lymph node from one of the patients contained fibrosing epithelioid granulomas consistent with the history of sarcoidosis (subject 6). Possibilities to explain this unusual combination of pulmonary findings include simultaneous occurrence of two diseases (sarcoidosis and idiopathic pulmonary fibrosis) or the development of significant chronic interstitial pneumonitis as an intrinsic component of late-stage sarcoidosis. With regard to the first of these possibilities, it would seem unlikely considering that these are two uncommon disorders of the lungs. As for the second possibility, it is well known that the "UIP pattern" is a histological pattern of injury that is not specific, and can be seen in several diseases, most commonly idiopathic pulmonary fibrosis, but also collagen vascular diseases, asbestosis and drug reactions. In addition, granulomatous 

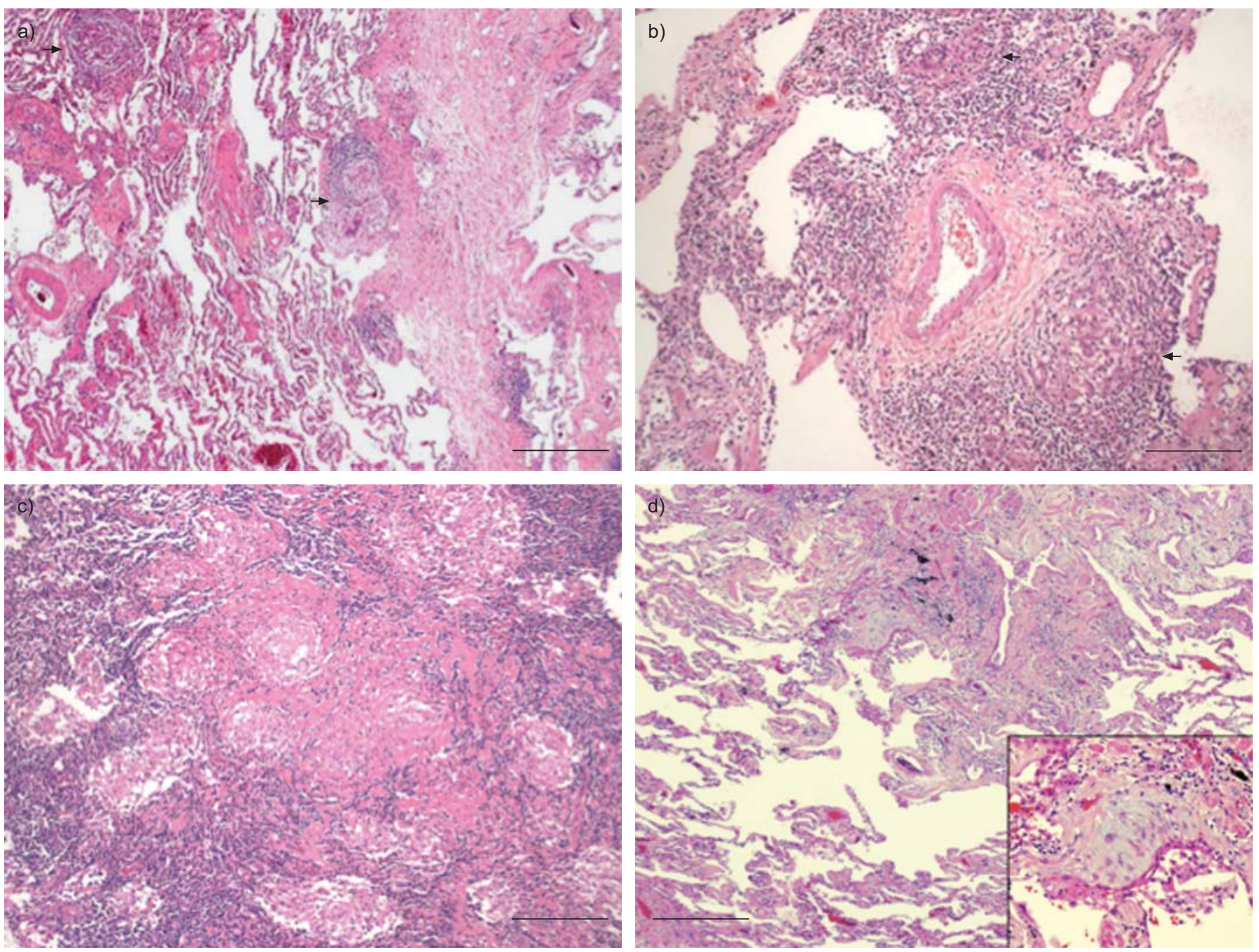

FIGURE 1. Histological findings (haematoxylin and eosin stain) from lung explants of sarcoidosis patients after lung transplantation in a) subject 2, b) subjects 4 and c d) subject 6. a) Granuloma formation (arrows) and fibrosis along the bronchovascular bundles and within the interstitium adjacent to otherwise normal appearing alveoli. b) Granuloma formation along bronchovascular bundle (arrows) with moderate-to-severe chronic interstitial pneumonitis. c) Fibrosing epithelioid granulomas from a regional lymph node. The lungs in this subject showed a usual interstitial pneumonitis pattern. d) Lung section showing a variegated pattern with severe fibrosis and fibroblastic foci adjacent to less involved parenchyma. The insert shows higher magnification of fibroblast focus. a) Scale bar $=300 \mu \mathrm{m}$. b) Scale bar $=100 \mu \mathrm{m}$. c, d) Scale bars $=200 \mu \mathrm{m}$.

diseases, namely chronic hypersensitivity pneumonitis, can produce a UIP pattern in late stages.

We also observed that subjects who did not have interstitial pneumonitis had sarcoidosis for a longer time prior to transplant (subjects 1-3; mean (range) 23.3 (17-33) yrs) compared to those who had evidence of interstitial pneumonitis (subjects 47; 4.8 (3-7) yrs). These data, although limited, suggest there may be a subset of sarcoidosis patients who show rapid progression of the disease, ultimately leading to lung transplantation. Further studies are warranted to support these observations and to elucidate the exact mechanisms that contribute to the clinical course of patients with sarcoidosis.

Our study may not be generalisable to all patients with endstage sarcoidosis since lung transplant recipients have usually undergone an extensive pre-transplant evaluation and this may limit the extrapolation of these findings. Our cases were referrals obtained from the community with a diagnosis of sarcoidosis established 3-22 yrs previously, making it difficult to obtain the original biopsy. However, five of the seven subjects at explant showed either mediastinal/hilar lymph nodes and/or lung tissues that still contained typical noncaseating epithelioid granulomas. These findings, combined with the radiographic and clinical history summarised in table 1, make the diagnosis of sarcoidosis more certain. Two out of the four patients with chronic interstitial pneumonitis who did not show residual granulomas had clinical history, radiographic patterns and surgical pathology reports of noncaseating granulomas on biopsies that were consistent with the diagnosis of sarcoidosis (subjects 5 and 7; table 1). In fact, in end-stage sarcoidosis it may be difficult to identify residual granulomatous inflammation $[2,10]$.

In conclusion, a subset of patients with end-stage sarcoidosis who underwent lung transplantation had severe chronic 


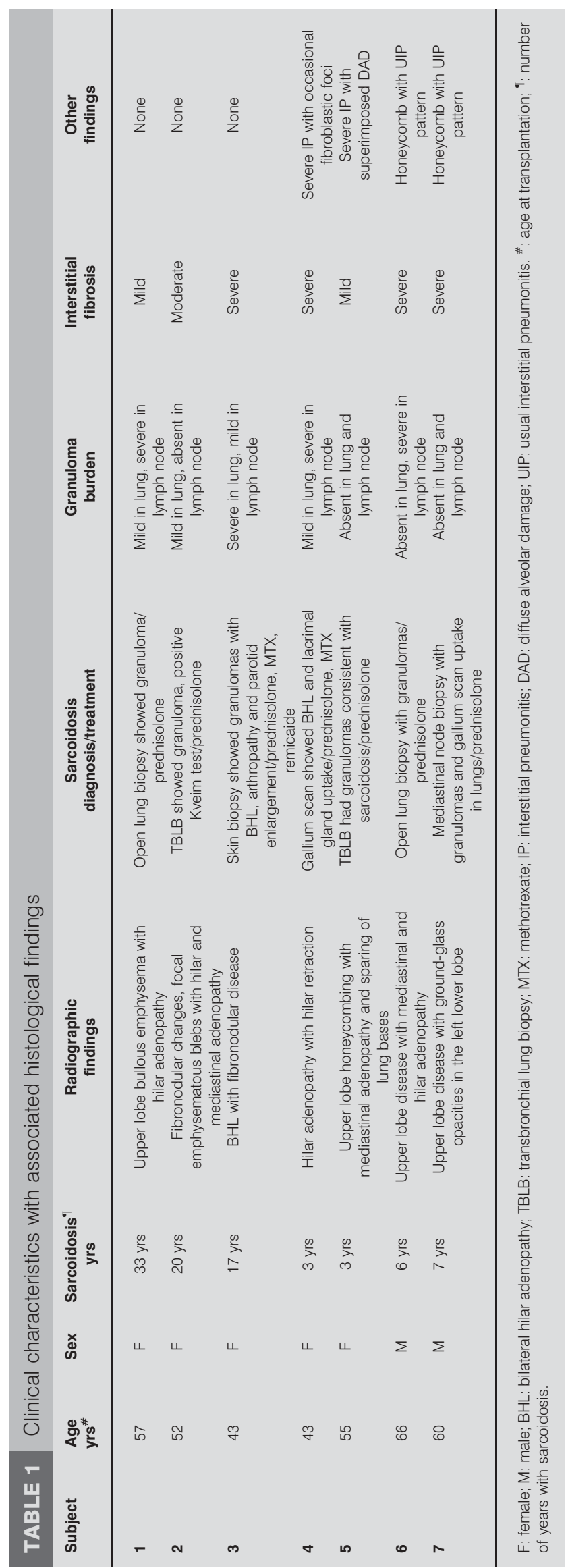

interstitial pneumonitis, in some cases with UIP pattern, hitherto considered atypical of end-stage sarcoidosis. The histological progression of sarcoidosis to end-stage fibrosis has not been fully investigated. Further studies are needed to elucidate the significance of chronic interstitial pneumonitits, especially UIP pattern, in end-stage sarcoidosis as a potential marker for progressive disease.

\section{H. Shigemitsu*, J.M. Oblad", O.P. Sharma* and M.N. Koss"} Depts of Pulmonary and Critical Care Medicine, and \#Pathology, University of Southern California, Keck School of Medicine, Los Angeles, CA, USA.

Correspondence: H. Shigemitsu, Dept of Pulmonary and Critical Care Medicine, Keck School of Medicine, University of Southern California, 1200 N State St, GNH 11-900, Los Angeles, CA, USA. E-mail: hshigemi@usc.edu

Statement of Interest: A statement of interest for O.P. Sharma can be found at www.erj.ersjournals.com/misc/statements.dtl

\section{REFERENCES}

1 Leslie KO, Colby TV, Swensen SJ. Anataomic distribution, histopathologic patterns of interstitial lung disease, In: Schwarz MI., King TE, eds. Interstitial Lung Disease. 4th Edn. Hamilton, Decker Inc, 2003; 40-41.

2 Sporn T, Roggli VL. Predominately mature interstitial fibrosis. In: Cagle PT., Allen TC., Beasley MB, eds. Diagnostic Pulmonary Pathology. 2nd Edn. London, Informa Health Care, 2008; 93-95.

3 Costabel U, Bross KJ, Guzman J, et al. Predictive value of bronchoalveolar T-cell subsets for the course of pulmonary sarcoidosis. Ann N Y Acad Sci 1986; 465: 418-426.

4 Crystal RG, Roberts WC, Hunninghake GW, et al. Pulmonary sarcoidosis: a disease characterized and perpetuated by activated lung T lymphocytes. Ann Intern Med 1981; 94: 73-94.

5 Mitchell DN, Scadding JD, Heard BE, et al. Sarcoidosis: histopathological definition and clinical diagnosis. J Clin Pathol 1976, 30: 395-408.

6 Hunninghake GW, Costabel U, Ando M, et al. ATS/ERS/WASOG Statement on Sarcoidosis. Am J Resp Crit Care Med 1999; 160: 736-755.

7 Rosen Y, Athanassiades TJ, Moon S, et al. Nongranulomatous interstitial pneumonitis in sarcoidosis. Relationship to development of epithelioid granulomas. Chest 1978; 74: 122-125.

8 Keogh BA, Hunninghake GW, Line BR. The alveolitis of pulmonary sarcoidosis: evaluation of natural history and alveolitis-dependent changes in lung function. Am Rev Respir Dis 1983; 128: 256-265.

9 Aisner SC, Albin RJ. Diffuse interstitial pneumonitis and fibrosis in sarcoidosis. Chest 1988; 94: 193-195.

10 Rosen Y. Sarcoidosis. In: Dail DH, Hammar SP, eds. Pulmonary Pathology. 1st Edn. New York, Springer-Verlag 1988; 427-430. 